\title{
Effect of GATA4 gene methylation on proliferation and apoptosis of SGC-7901 gastric cancer cells
}

\author{
X. MA ${ }^{1, \star}$, L. JI ${ }^{2}$ \\ ${ }^{1}$ Department of Gastroenterology, Shaanxi Provincial People's Hospital, Xian, 710068, China; ${ }^{2}$ Department of Orthopedic Surgery, Shaanxi \\ Provincial People's Hospital, Xi'an, 710068, China \\ ${ }^{*}$ Correspondence: maxiaoying19@163.com
}

Received April 11, 2019 / Accepted September 9, 2019

\begin{abstract}
Gastric cancer (GC) remains a major cause of cancer-related deaths worldwide. GATA4 has been previously reported to exhibit functions in GC. In this study, we aimed to investigate the effect of GATA4 gene methylation on GC progression. Methylation-Sensitive High-Resolution Melting was used to detect the methylation of GATA4 promoter region in GC tissues and adjacent normal tissues, GC cell lines and GES-1 cells. The relationship between GATA4 methylation level and clinical characteristics of GC patients was analyzed. GATA4 levels in GC tissues and adjacent normal tissues, GC cell lines and GES-1 cell lines were detected, and gain-of-function was performed to investigate the role of GATA4 in GC. si-GATA4 was transfected into GES-1 cells to observe the changes of various indicators. RNA-seq detected the differentially expressed genes in SGC-7901 cells overexpressing GATA4, and western blot analysis verified their expression. GATA4 methylation rate was increased in GC tissues, and GATA4 promoter was abnormally methylated in GC cells. GATA4 methylation rate in GC tissues was related to lymph node metastasis, differentiation degree and clinical stage of GC patients. Lower expressed GATA4 was observed in GC tissues and cells. Cell proliferation rate decreased and cell apoptosis rate increased in SGC-7901 cells overexpressing GATA4. Transfecting si-GATA4 into GES-1 cells led to increased proliferation, inhibited apoptosis, and restoration of GATA4 led to decreased APC and GSK3 $\beta$ levels. In conclusion, restoration of GATA4 caused by 5-Aza treatment can inhibit the proliferation and promote apoptosis of GC cells possibly via Wnt/ $\beta$-catenin signaling pathway. This study may offer new sight for GC treatment.
\end{abstract}

Key words: gastric cancer, GATA4, promoter methylation, proliferation, apoptosis

Gastric cancer (GC) is estimated to be the fifth most frequently diagnosed cancer worldwide and it ranks third in regard to cancer-related deaths in both males and females all over the world [1]. It has been observed that over $70 \%$ of new cases and deaths occur in developing regions and countries [2]. A research made in 2012 found that GC incidence rate in males was nearly two times higher than that in females globally, and an increasing trend that corresponded with increasing age, peaking at 80-84 years, as well as increased median and the average age [3]. It is commonly believed that the incidence and mortality of GC are associated with the economic status and diet, mainly environmental sanitation for water and housing, Helicobacter pylori infection, high intake of salt, pickled or smoked foods, as well as refined carbohydrates [4]. The prognosis is far from satisfaction since its average 5-year survival rate is less than $20 \%$ owing to late diagnosis [5]. In recent years, although diverse molecules have been utilized for GC prediction, their roles in identifying the individual risk are quite limited [6]. Therefore, there is an urgent need to find new diagnostic markers to prevent GC incidence and improve the overall treatment effect of GC patients.

GATA transcription factors are zinc finger DNA-binding proteins, which exert functions in the development of various tissues by activating or inhibiting transcription, thus regulating the proliferation, differentiation, and cell survival, and their reduced expression can lead to malignant transformation $[7,8]$. GATA4, a member of the GATA family, can regulate gene expression and play a crucial role in cardiac development, gastrointestinal system, respiratory system as well as cancer [9]. It has been previously reported that GATA4 displays genomic amplification in GC, and the combination of KLF5, GATA4, and GATA6 promotes GC development [10]. It has previously been shown that the GATA4 gene methylation is related to poor lung function and increased odds of chronic obstructive pulmonary disease, and showed 
a 3-fold increase in the risk of poor health [11]. GATA4 methylation has also been reported to play an important role in epithelial ovarian cancer [12], and the methylation level of GATA4/5 has been considered as a noninvasive biomarker of colorectal cancer [13]. Based on the above literature, we can draw a hypothesis that GATA4 is involved in GC through its gene promoter methylation. Therefore, this study aims to explore the effects of GATA4 on proliferation and apoptosis in GC cells.

\section{Materials and methods}

Sample collection. From September 2016 to September 2018, 64 GC patients (46 males and 18 females, with a median age of 36-77 years and an average age of $59.5 \pm 10.4$ years) diagnosed and treated in Shaanxi Provincial People's Hospital were enrolled in this experiment for collection of GC tissues and adjacent normal tissues (at least $5 \mathrm{~cm}$ away from GC tissues). Inclusion criteria were as follows: 1) all patients were diagnosed as GC by pathological examination after operation; 2) no patients received radiotherapy or chemotherapy before operation; 3) all patients had complete clinical data. Patients with chronic system diseases or other malignant tumors would be excluded. This study was approved by and supervised by the ethics committee of Shaanxi Provincial People's Hospital. All the subjects signed the informed consent.

GC cell selection and cultivation. GC cell lines (BGC-823, AGS, and SGC-7901) purchased from the Cell Resource Center of Shanghai Institute of Biology, Chinese Academy of Sciences (Shanghai, China) and human normal gastric mucosal cell line GES-1 purchased from Shanghai Aiyan Biological Technology Co., Ltd. (Shanghai, China) were cultured with medium (Gibco, Grand Island, NY, USA) containing $10 \%$ fetal bovine serum (FBS) and $1 \%$ penicillin streptomycin in a $5 \% \mathrm{CO}_{2}$ incubator at $37^{\circ} \mathrm{C}$. When cell density reached about $80 \%$, the cells were subcultured into the second or third generation. Cells in logarithmic growth

Table 1. Primer sequences of methylation.

\begin{tabular}{ll}
\hline Gene & Primer \\
\hline \multirow{2}{*}{ GATA4 } & F: 5'-GGTTGAAGTGTAGTGGTATGATTTT-3' \\
& R: 5'-ATTCAACACCAAACTAACCAACATA-3' \\
\hline
\end{tabular}

Table 2. Primer sequences of RT-qPCR.

\begin{tabular}{ll}
\hline Genes & Sequences \\
\hline \multirow{2}{*}{ GATA4 } & F: 5'-ATAAAGCTGACCC TGGGCAC-3' \\
& R: 5'-GGGTGAATTCAGCTGCTCCT-3' \\
\multirow{2}{*}{ GAPDH } & F: 5'-TGGGTGTGAACCATGAGAAG-3' \\
& R: 5'-GTGTCGCTGTTGAAGTCAGA-3' \\
\hline
\end{tabular}

Note: GAPDH: Glyceraldehyde-3-phosphate dehydrogenase; RT-qPCR: reverse-transcription quantitative polymerase chain reaction; $\mathrm{F}$, forward; $\mathrm{R}$, reverse. phase were selected to detect the expression of GATA4 and the methylation of $\mathrm{CpG}$ island in the promoter region. SGC-7901 cells with high methylation level were selected for demethylation treatment and GATA4 overexpression experiments.

SGC-7901 cells were cultured in Dulbecco's modified Eagles medium (DMEM) containing 10\% FBS and treated with 1\% 5-aza-cytidine (Sigma, St. Louis, MO, USA) dissolved in dimethyl sulphoxide (DMSO) for $72 \mathrm{~h}$. Finally, the methylation level and expression of GATA4 in SGC-7901 cells were detected.

Methylation-Sensitive High-Resolution Melting (MS-HRM). The genomic DNA of tissues and cells was extracted by conventional phenol-chloroform extraction and transformed by EpiTect fast DNA Bisulfite kit. The reaction system included $1000 \mathrm{ng}$ DNA, $35 \mu \mathrm{l}$ DNA protective solution and $85 \mu \mathrm{l}$ conversion reagent. The reaction condition was at $95^{\circ} \mathrm{C}$ for $5 \mathrm{~min}$, at $60^{\circ} \mathrm{C}$ for $20 \mathrm{~min}$, at $95^{\circ} \mathrm{C}$ for $5 \mathrm{~min}$, and at $60^{\circ} \mathrm{C}$ for $20 \mathrm{~min}$. After the reaction, the product was purified and recovered with a centrifugal column attached to the kit and then stored at $-20^{\circ} \mathrm{C}$. MS-HRM was carried out on Rotor-gene 6000 (Corbett, Sydney, Australia) in two copies of each sample. Each analysis included $100 \%, 50 \%, 10 \%$, and $3 \%$ methylated and completely unmethylated standard samples for the detection of methylation levels. MS-HRM primers were designed and synthesized by Shanghai Sangon Biotechnology Co., Ltd. Shanghai, China (Table 1). The $20 \mu \mathrm{l}$ reaction system consisted of $1 \times$ PCR buffer, 1.5 to $3.0 \mathrm{mM}$ $\mathrm{MgCl}_{2}, 200 \mu \mathrm{M}$ dNTP mixture, $200-400 \mathrm{nM}$ forward and reverse primers, $1 \times$ SYTO9 intercalating dye (Thermo Fisher Science, Waltham, MA, USA), 0.5 U Hotstar Taq polymerase and $10 \mathrm{ng}$ hydrogen sulfite-transformed DNA. The methylation level was presented as a percentage.

GC cell grouping and treatment. SGC-7901 cells in the logarithmic growth phase were allocated into a blank group (without any treatment), negative control (NC) group (transfected with empty vector pcDNA3.1), GATA4 group (transfected with pcDNA-GATA4 plasmid, overexpressed GATA4 plasmid constructed by pcDNA3.1 vector), si-NC group and si-GATA4 group. The empty vector pcDNA3.1, pcDNAGATA4 plasmid, and si-NC were synthesized by Shanghai Sangon Biotechnology Co., Ltd. (Shanghai, China). Transient transfection was carried out strictly according to the instructions of Lipofectamine 2000 (Invitrogen, Carlsbad, CA, USA). After $48 \mathrm{~h}$, the mRNA expression and protein level of GATA4 in cells were detected by reverse-transcription quantitative polymerase chain reaction (RT-qPCR) and western blot analysis to verify the transfection effect.

RT-qPCR. Trizol (Invitrogen, Carlsbad, CA, USA) one-step method was used to extract total RNA of tissues and cells, and the extracted high quality RNA was confirmed by ultraviolet analysis and formaldehyde denaturation electrophoresis. One $\mu \mathrm{g}$ RNA was reversely transcribed into cDNA by avian myeloblastosis virus (AMV) reverse transcriptase. The qPCR was conducted by SYBR Green method with 
glyceraldehyde-3-phosphate dehydrogenase (GAPDH) as an internal reference. The PCR primers were designed and synthesized by Shanghai Sangon Biotechnology Co., Ltd., Shanghai, China (Table 2). PCR system included cDNA $1.0 \mu \mathrm{l}, 2 \times$ SYBR Green Mix $10 \mu \mathrm{l}$, Forward Primer $(10 \mu \mathrm{M})$ $0.5 \mu \mathrm{l}$, Reverse Primer $(10 \mu \mathrm{M}) 0.5 \mu \mathrm{l}$, and was supplemented into $20 \mu \mathrm{l}$ with the addition of RNase free water. The reaction conditions were as follows: pre-denaturation at $94^{\circ} \mathrm{C}$ for $5 \mathrm{~min}, 40$ cycles of denaturation at $94^{\circ} \mathrm{C}$ for $40 \mathrm{~s}$, annealing at $60^{\circ} \mathrm{C}$ for $40 \mathrm{~s}$, extension at $72^{\circ} \mathrm{C}$ for $60 \mathrm{~s}$, and extension at $72^{\circ} \mathrm{C}$ for $10 \mathrm{~min}$ at last. The products were verified by agarose gel electrophoresis. The threshold value was selected manually at the lowest point of the parallel rise of logarithmic amplification curves, and the threshold cycle $(\mathrm{Ct})$ value of each reaction tube was obtained. The data were analyzed by $2^{-\Delta \Delta C t}$ method, which indicated the multiple relationships between the experimental group and the control group. The formula was as follows: $\Delta \Delta \mathrm{Ct}=[\mathrm{Ct}$ (target gene) $-\mathrm{Ct}$ (reference gene) $]_{\text {experimental group }}-[\mathrm{Ct}$ (target gene) $-\mathrm{Ct}$ (reference gene)] control group.

Western blot analysis. The protein concentration of each tissue and cell was determined according to the instructions of bicinchoninic acid (BCA) kit (Boster Biological Technology Co., Ltd, Wuhan, Hubei, China). The extracted protein was added with the loading buffer, then boiled at $95^{\circ} \mathrm{C}$ for $10 \mathrm{~min}$, and loaded on each well (each for $30 \mu \mathrm{g}$ ), and then protein was separated by $10 \%$ polyacrylamide gel electrophoresis (PAGE, Boster Biological Technology) with the voltage changing from $80 \mathrm{~V}$ to $120 \mathrm{~V}$. The protein was transformed into the polyvinylidene fluoride (PVDF) membrane by wet transformation at $100 \mathrm{mV}$ for $45-70$ min. The membrane was sealed in $5 \%$ bovine serum albumin (BSA) at room temperature for $1 \mathrm{~h}$ and added with the primary antibodies at $4^{\circ} \mathrm{C}$ overnight. Afterward, the membrane was rinsed by Tris-buffed saline containing $0.05 \%$ Tween 20 (TBST) for 3 times, each for $5 \mathrm{~min}$, and then incubated with the secondary antibody (ZSGB-Bio Co., Ltd, Beijing, China) at room temperature for $1 \mathrm{~h}$. Then the membrane was washed $3 \times 5 \mathrm{~min}$, developed by chemiluminescence reagent and bands were visualized using the Bio-Rad Gel Dol EZ imager (Bio-Rad Laboratories, Hercules, CA, USA). The target band was analyzed by ImageJ software (National Institutes of Health, Bethesda, Maryland, USA) for grey value analysis. All antibodies GATA4 (1:2000, ab227512), $\beta$-actin (1:5000, ab227387), APC (1:1000, ab15270), GSK3 $\beta$ (1:1000, ab93926), APOB (1:1000, ab20737) COX-2 (1:1000, ab15191) were purchased from Abcam (Cambridge, MA, USA).

Cell counting kit-8 (CCK-8). SGC-7901 cell suspension was diluted to a certain concentration and then inoculated in a 96-well plate with a density of $2 \times 10^{3} / 100 \mu \mathrm{l} /$ well, with 12 parallel wells in each group. The cells were cultivated for $24 \mathrm{~h}, 48 \mathrm{~h}, 72 \mathrm{~h}$, and $96 \mathrm{~h}$, with 3 duplicated wells at each time point. CCK-8 solution (Sigma, St. Louis, MO, USA) was added to the medium without cells and served as the blank control. The plate was then cultured at $37^{\circ} \mathrm{C}$ with $5 \% \mathrm{CO}_{2}$. A total of $10 \mu \mathrm{l}$ CCK- 8 solution was added to the wells at each time point, and the plate was cultured in an incubator for $4 \mathrm{~h}$. The optical density (OD) value was detected at a wavelength of $450 \mathrm{~nm}$ by a microplate reader.

5-ethynyl-2'-deoxyuridine (EdU) assay. Cell-light EdU fluorescence detection kit (RiboBio, Guangzhou, Guangdong, China) was used to detect the DNA replication ability of cells. SGC-7901 cells in each group were resuspended and counted after routine treatment. The cells were inoculated into a 96-well plate at $1 \times 10^{4}$ cells/well, and each group had 3 duplicated wells. After $2 \mathrm{~h}$ cultivation in $100 \mu \mathrm{l} 50 \mu \mathrm{M}$ EdU, the cells were rinsed by phosphate buffered saline (PBS) twice, fixed with $4 \%$ paraformaldehyde for $20 \mathrm{~min}$, incubated with $2 \%$ glycine for $15 \mathrm{~min}$, and washed twice by PBS with the liquid absorbed. Then the cells were treated with $150 \mu \mathrm{l}$ penetrating agent and washed repeatedly by PBS for 3 times. The cells were treated according to the instructions of the EdU kit. Under the fluorescence microscope (Olympus FSX100), five visual fields were randomly photographed. Blue fluorescence represented all cells, while red fluorescence represented the replicating cells infiltrated by EdU. The percentage of EdU positive cells was then calculated.

Colony formation assay. SGC-7901 cells in each group were treated with trypsin, then the cell suspension was fully dispersed, and 200 cells were inoculated into a 6-well plate. The plate was shaken gently so that the cells were evenly dispersed and cultured for 2-3 weeks. When cell clones were visible to the naked eyes, the cultivation was terminated, the culture medium was removed, and cells were washed 3 times with PBS. Afterward, cells were fixed with $4 \%$ paraformaldehyde for $30 \mathrm{~min}$ and washed with PBS 3 times again. Subsequently, cells were stained with Giemsa solution for $60 \mathrm{~min}$, and the dying solution was washed away slowly by running water and cells were dried. Finally, the number of cell clones was counted under the microscope.

Flow cytometry. After treatment, suspended cells were mixed, centrifuged and collected with supernatant discarded. Cells were resuspended, washed with PBS and the concentration was adjusted to $1 \times 10^{6} \mathrm{cells} / \mathrm{ml}$ to reach dispersed single cell suspension. The cells were centrifuged at $2000 \mathrm{rpm}$ for $5 \mathrm{~min}$ and the supernatant was removed. Afterward, cells in each group were added with $500 \mu \mathrm{l}$ cold ethanol with a volume fraction of about $70 \%$ and fixed for $2 \mathrm{~h}$ to overnight at $4{ }^{\circ} \mathrm{C}$. After discarding the fixative liquid, $1 \mathrm{ml}$ PBS was added to further elute the fixative liquid. Cells were centrifuged at $2000 \mathrm{rpm}$ for $3 \mathrm{~min}$ with the supernatant removed, added with $100 \mu \mathrm{l}$ RNase $\mathrm{A}$ in a water bath at $37^{\circ} \mathrm{C}$ for $30 \mathrm{~min}$, and stained with $400 \mu \mathrm{l}$ propidium iodide (PI) at $4^{\circ} \mathrm{C}$ for $30 \mathrm{~min}$ in the dark. Subsequently, the red fluorescence at $488 \mathrm{~nm}$ excitation wavelength was recorded.

After treatment, cells were centrifuged with supernatant discarded. Cells were resuspended and washed with PBS and the concentration was adjusted into $1 \times 10^{6}$ cells $/ \mathrm{ml}$. A total of $200 \mu \mathrm{l}$ cells were centrifuged after twice wash by $1 \mathrm{ml}$ 
pre-cooled PBS. Cells were re-suspended in $100 \mu \mathrm{l}$ binding buffer, added with $2 \mu \mathrm{L}$ Annexin-V- fluorescein isothiocyanate (FITC) $(20 \mu \mathrm{g} / \mathrm{ml})$, mixed gently and placed on the ice for $15 \mathrm{~min}$ avoiding exposure to light. Then the cells were transferred to the flow detection tube and added with $300 \mu \mathrm{l}$ PBS. Each sample was added with $1 \mu \mathrm{l}$ PI $(50 \mu \mathrm{g} / \mathrm{ml})$ before detection and tested within $30 \mathrm{~min}$. With Annexin- $\mathrm{V}$ as the horizontal axis, PI as the vertical axis, mechanically damaged cells were located in the upper left quadrant, apoptotic or necrotic cells in the upper right quadrant, negative normal cells in the left lower quadrant and early apoptotic cells in the right lower quadrant.

Hoechst 33258 staining. SGC-7901 cells in the logarithmic growth phase were inoculated at $1 \times 10^{5}$ cells $/ \mathrm{ml}$ into a 6-well plate with a cover glass, $3 \mathrm{ml}$ per well. After cells were cultivated in a $5 \% \mathrm{CO}_{2}$ incubator at $37^{\circ} \mathrm{C}$ for $24 \mathrm{~h}$, the culture medium was removed. The fixative solution was removed after cells were fixed for $10 \mathrm{~min}$, and then the Hoechst 33258 dyeing (Shanghai Beyotime Biotechnology Co., Ltd, Shanghai, China) was added following cell washing. Cells were stained at room temperature for $5 \mathrm{~min}$, and then the dyeing solution was removed and cells were washed with PBS. The cover glass attached with cells was taken out and placed on a slide dripped with an anti-fluorescence quenching agent. The slide was observed and photographed under a fluorescence microscope.

Transwell assay. Cell migration experiments were carried out in a Transwell chamber without polyvinylpyrrolidone polyvinyl acetate membrane with well size of $8 \mu \mathrm{m}$. GES-1

Table 3. The relationship between the degree of GATA4 methylation and the clinical characteristics of GC patients.

\begin{tabular}{lcccc}
\hline \multirow{2}{*}{$\begin{array}{l}\text { Clinicopathological } \\
\text { data }\end{array}$} & Cases & \multicolumn{2}{c}{ GATA4 methylation } & \\
\cline { 3 - 4 } & & $\begin{array}{c}\text { Positive } \\
(\mathbf{n}=39)\end{array}$ & $\begin{array}{c}\text { Negative } \\
(\mathbf{n}=25)\end{array}$ & p-value \\
\hline Age & & & & 0.308 \\
$\quad<60$ & 30 & $16(53.3)$ & $14(46.7)$ & \\
$\quad 360$ & 34 & $23(67.6)$ & $11(32.4)$ & \\
$\quad$ Sex & & & & 0.273 \\
$\quad$ Male & 46 & $26(56.5)$ & $20(43.5)$ & \\
$\quad$ Female & 18 & $13(72.2)$ & $5(27.8)$ & \\
Tumor size & & & & 0.193 \\
$\quad \leq 5 \mathrm{~cm}$ & 26 & $13(50.0)$ & $13(50.0)$ & \\
$\quad>5 \mathrm{~cm}$ & 38 & $26(68.4)$ & $12(31.6)$ & \\
Clinical stages & & & & 0.010 \\
$\quad$ I+II & 35 & $16(45.7)$ & $19(54.3)$ & \\
$\quad$ III+IV & 29 & $23(79.3)$ & $6(20.7)$ & \\
Lymph node metastasis & & & & 0.015 \\
$\quad$ Yes & 41 & $30(73.2)$ & $11(26.8)$ & \\
$\quad$ No & 23 & $9(39.1)$ & $14(60.9)$ & \\
$\begin{array}{l}\text { Degree of differentiation } \\
\quad\end{array}$ & & & & 0.019 \\
$\quad \begin{array}{l}\text { Poor + moderate } \\
\text { High }\end{array}$ & 47 & $33(70.2)$ & $14(29.8)$ & \\
\hline
\end{tabular}

Note: GC, gastric cancer. Data were analyzed by Fisher's exact test. cells transfected with $1 \times 10^{5}$ cells $/ \mathrm{ml}$ siRNA was placed in the upper chamber with serum medium $(0.1 \% \mathrm{BSA})$ and in the lower chamber with DMEM containing 10\% FBS. After cultivation in a $5 \% \mathrm{CO}_{2}$ incubator at $37^{\circ} \mathrm{C}$ for $24 \mathrm{~h}$, the filtration membrane was taken out and washed with PBS, fixed with $0.5 \%$ glutaraldehyde, stained with crystal violet staining solution at $37^{\circ} \mathrm{C}$ in a $5 \% \mathrm{CO}_{2}$ incubator. Five visual fields (200x) were randomly counted under the microscope.

Total RNA was extracted from cells by miRNAeasy Mini Kit (Qiagen, Dusseldorf, Germany), quantified by NanoDrop ND-2000 spectrophotometer (Thermo Fisher Scientific), and differentially expressed RNA was screened by microarray analysis (Sangon Biotech, Shanghai, China). The threshold was set at a multiple of more than 2 , and $\mathrm{p}<0.05$.

Statistical analysis. SPSS 21.0 (IBM Corp., Armonk, NY, USA) was applied for data analysis. Kolmogorov-Smirnov test showed whether the data were in the normal distribution. The results were expressed as mean \pm standard deviation. Comparison between the two groups was analyzed by t-test, comparison among multiple groups was analyzed by one-way analysis of variance (ANOVA) or two-way ANOVA, and pairwise comparison after ANOVA was conducted by Tukey's multiple comparisons test. Fisher's exact test was used to compare the enumeration data. $p$ was obtained by a two-tailed test and $\mathrm{p}<0.05$ indicated a significant difference.

\section{Results}

Hypermethylation of GATA4 in GC tissues and cells. The methylation of GATA4 promoter region in GC tissues was detected by MS-HRM. The results showed that the methylation rate of the GATA4 promoter region in GC tissues was significantly higher than that in adjacent tissues (Figure 1). Then we detected the methylation of the GATA4 promoter region in GC cell lines BGC-823, AGS, SGC-7901, and human normal gastric mucosa cell line GES-1. The results showed that no promoter methylation of the GATA4 gene was detected in human normal gastric mucosal cell line GES-1, while there was significant abnormal methylation in the promoter region of the GATA4 gene in GC cell line GES-1 (Figure 1). This indicated that there was abnormal methylation of GATA4 in GC.

GATA4 methylation is associated with the clinical stage and lymph node metastasis of GC patients. The relationship between the GATA4 methylation level and the clinical characteristics of GC patients was analyzed. The results showed that the GATA4 methylation level in GC tissues was related to the clinical stage, lymph node metastasis, and tumor differentiation. The methylation rate of GATA4 increased with the increase of GC clinical stages. The methylation rate of GATA4 in GC tissues of patients in stage III+IV was significantly higher than that of patients in stage I+II $(\mathrm{p}<0.05)$. The methylation rate of GATA4 in poorly differ- 

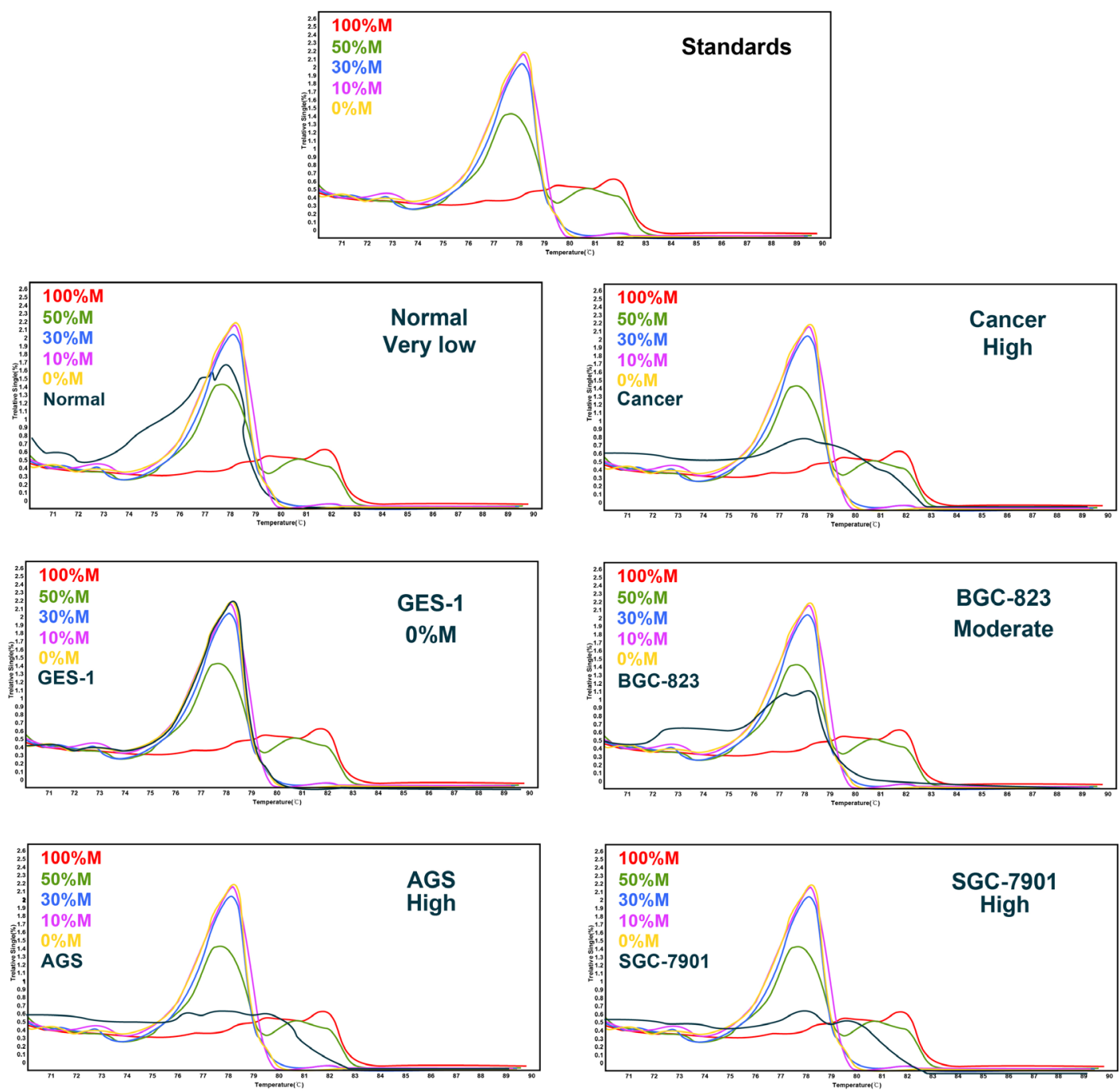

Figure 1. GATA4 is hypermethylated in GC tissues and cells. MS-HRM was used to detect the degree of GATA4 methylation in tissue specimens and cell lines. $0 \% \mathrm{M}$ was non-methylation, $\mathrm{N}=64$, Repetitions = 3. GC, gastric cancer; MS-HRM, methylation-sensitive high-resolution melting.

entiated GC tissues was significantly higher than that in highly differentiated GC tissues $(\mathrm{p}<0.05)$. The methylation rate of GATA4 in GC tissues with lymph node metastasis was significantly higher than that in GC tissues without lymph node metastasis $(p<0.05)$. There was no significant correlation between GATA4 methylation and age, sex, and tumor size (all $\mathrm{p}>0.05$; Table 3 ).

GATA4 is poorly expressed in GC tissues and cells. RT-qPCR and western blot analysis were used to detect the mRNA and protein levels of GATA4 in GC tissues and adjacent normal tissues as well as in GC cell lines BGC-823, AGS, SGC-7901, and human normal gastric mucosa cell line GES-1. The results showed that the mRNA and protein levels of GATA4 in GC tissues were significantly lower than those in adjacent normal tissues ( $<<0.05$; Figure $2 \mathrm{~A}$ ). Besides, the mRNA and protein levels of GATA4 in GC cell lines BGC-823, AGS, and SGC-7901 were significantly lower than those in normal gastric mucosa cell line GES-1 (all $\mathrm{p}<0.05$; Figure 2B).

SGC-7901 cells with a high level of methylation were selected and demethylated with 5-Aza. Then the promoter methylation of GATA4 was detected by MS-HRM. It was found that the methylation level of the GATA4 promoter in SGC-7901 cells decreased significantly after demethylation, while the level of GATA4 increased significantly (Figures 2C and $2 \mathrm{D}$; all $\mathrm{p}<0.05)$. These results suggested that the level of GATA4 in GC was regulated by the methylation of its promoter region, and the abnormal methylation of GATA4 led to the low level of GATA4.

Restoration of GATA4 inhibits the proliferation of SGC-7901 cells. To construct SGC-7901 cell line with stable overexpression of GATA4, the overexpression plasmid of GATA4 was transfected into SGC-7901 cells by Lipofectamine 2000. After $48 \mathrm{~h}$ of transfection, the level of 

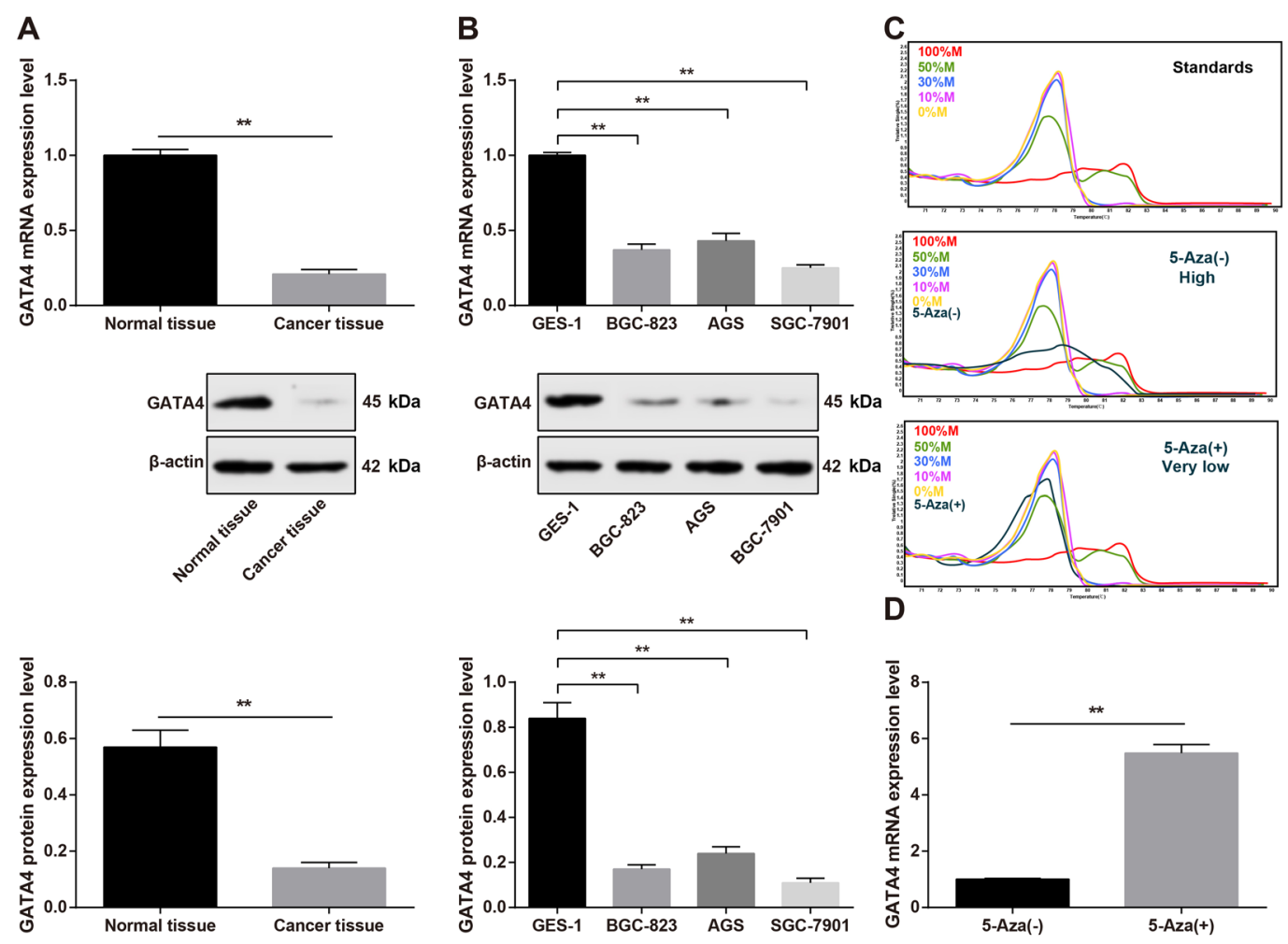

Figure 2. GATA4 is poorly expressed in GC tissues and cells. A) The mRNA and protein levels of GATA4 in GC and adjacent normal tissues were detected by RT-qPCR and Western blot analysis, $\mathrm{N}=64$; B) The mRNA and protein levels of GATA4 in GC cells and human normal gastric mucosa cells GES-1 were detected by RT-qPCR and western blot analysis; C) The GATA4 methylation in SGC-7901 cells after demethylation was detected by MS-HRM; D) The level of GATA4 in demethylated cells was detected by RT-qPCR; Repetitions = 3; One-way ANOVA was used to analyze the data, and Tukey's multiple comparisons test was used for pairwise comparisons after ANOVA; ${ }^{\star \star} \mathbf{p}<0.01$. GC, gastric cancer; RT-qPCR, reverse-transcription quantitative polymerase chain reaction; MS-HRM, methylation-sensitive high-resolution melting; ANOVA, variance of analysis.

GATA4 was significantly increased (Figure 3A; all $\mathrm{p}<0.05$ ), indicating that the SGC-7901 cell line overexpressing GATA4 was successfully constructed.

In order to examine the role of GATA4 in GC cell proliferation, CCK-8 assay was performed. The results showed that there was no significant difference in cell proliferation rate between the NC and blank groups (all $p>0.05$ ). Cell viability in the GATA4 group was significantly lower than that of the blank and NC groups at $48 \mathrm{~h}, 72 \mathrm{~h}$, and $96 \mathrm{~h}$ with decreased cell proliferation rate (all $\mathrm{p}<0.05$; Figure $3 \mathrm{~B}$ ).

EdU assay was used to detect the DNA replication activity of SGC-7901 cells. The results showed that the DNA replication activity in the blank and $\mathrm{NC}$ groups was similar, showing no significant difference in the rate of EdU positive cells $(p>0.05)$. However, after stable transfection of the GATA4 overexpression plasmid, the DNA replication activity of SGC-7901 cells decreased significantly, showing that the rate of EdU positive cells decreased significantly (all $\mathrm{p}<0.05$; Figure 3C).

The colony formation ability of SGC-7901 cells in each group was tested by colony formation assay. The results showed that there was no significant difference in the number of clones between the blank and NC groups (all $\mathrm{p}>0.05$ ); compared with the blank group, the number of clones in the GATA4 group decreased significantly (all $\mathrm{p}<0.05$; Figure 3D). These results suggested that overexpression of GATA4 could inhibit the proliferation of GC cells.

Restoration of GATA4 inhibits SGC-7901 cell cycle and promotes apoptosis. Flow cytometry was used to detect the effect of overexpressed GATA4 on cell cycle distribution of SGC-7901 cells. There was no significant difference in cell proportion between the blank and NC groups ( $\mathrm{p}>0.05)$. Compared with the blank group, the number of G0/G1 cells in the GATA4 group increased significantly, while the number of $S$ and $\mathrm{G} 2 / \mathrm{M}$ cells decreased significantly (all $\mathrm{p}<0.05$; Figure $4 \mathrm{~A}$ ). These results suggested that overexpression of GATA4 arrested SGC-7901 cells in G0/G1 stage, and upregulation of GATA4 could inhibit the cell cycle of GC cells.

Morphological changes of apoptotic cells were observed by Hoechst 33258 staining combined with fluorescence microscopy. The nuclei of normal cells were round, clear edge and stained evenly; while the nuclei of apoptotic cells were irregular, chromatin agglutinated and stained heavily, 
with nuclear pyknosis and increased fragments of nucleosomes; the number of apoptotic cells in the GATA4 group was significantly more than that those in the blank and NC groups (Figure 4B).

Flow cytometry was used to detect the apoptotic rate of SGC-7901 cells in each group. The results showed that the apoptotic rate of SGC-7901 cells in the GATA4 group was significantly higher than that in the blank and NC groups $(\mathrm{p}<0.05$; Figure $4 \mathrm{C})$, indicating that the restoration of GATA4 expression could induce the apoptosis of SGC-7901 cells.

Low expression of GATA4 in GES-1 cells promotes cell migration and increases cell apoptosis. Compared with GC cells, GES-1 cells have a higher expression of GATA4. We transfected GES-1 cells with si-GATA4 and si-NC to further confirm the function of GATA4 in GC. Firstly, we designed and synthesized two si-GATA4. The expression of GATA4 decreased after transfection confirmed by RT-qPCR and western blot analysis. The si-GATA4-1 with a better interference effect was selected to carry out subsequent experiments (Figures 5A and 5B). Cell viability test showed that after the transfection of si-GATA4, cell viability increased significantly (Figure 5C). Cell cycle analysis showed that G0/G1 phase shortened, while $S$ phase and $G 2 / M$ phase extended (Figure 5D). GATA4 knockout significantly reduced the apoptosis of GES-1 cells (Figure 5E). Compared with the control group, the low expression of GATA4 significantly increased cell colonies (Figure 5F), and migration (Figure 5G). These results suggest that GATA4 is a tumor suppressor in gastric cancer.

Overexpression of GATA4 is a tumor suppressor in GC by inhibiting Wnt/ $\beta$-catenin signaling pathway. RNA-seq analysis was applied to detect the gene expression changes in SGC-7901 after overexpression of GATA4. We found that after overexpression of GATA4, the expression of many genes changed, among which the differentially expressed APC and GSK3 $\beta$, which are related factors of $\mathrm{Wnt} / \beta$-catenin signaling pathway, attracted our attention (Figure 6A). Then western blot analysis verified that levels of APC and GSK3 $\beta$ in cells after overexpression of GATA4 were significantly downregulated, consistent with the trend of RNA-seq
A

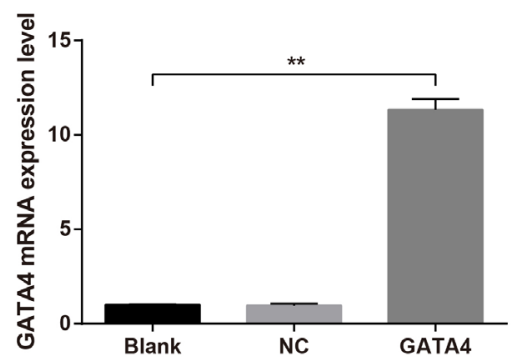

C
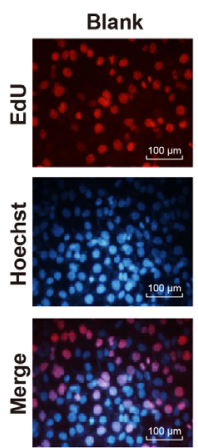
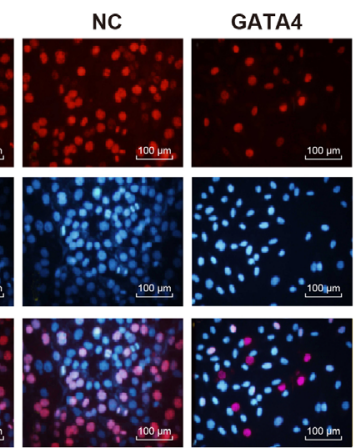
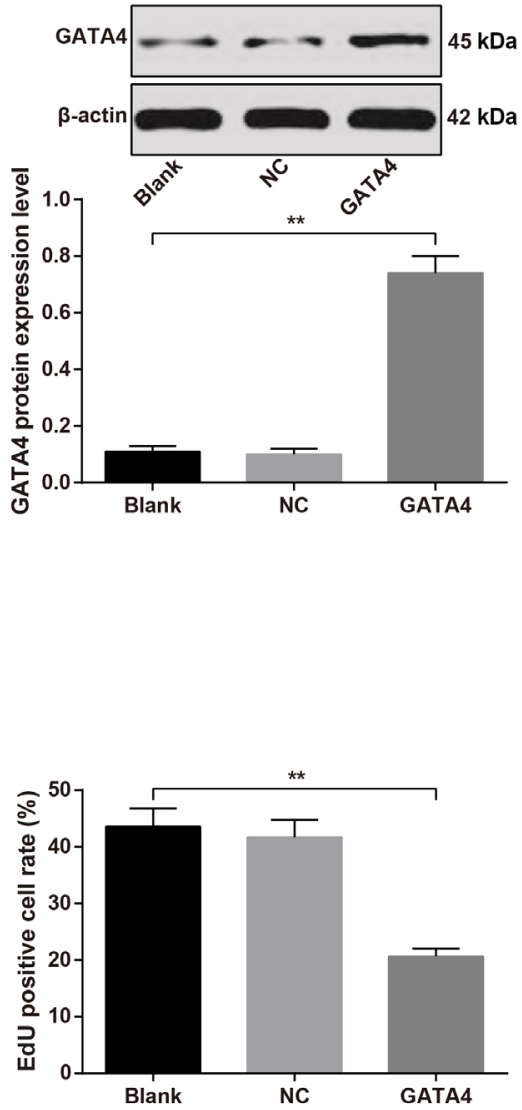

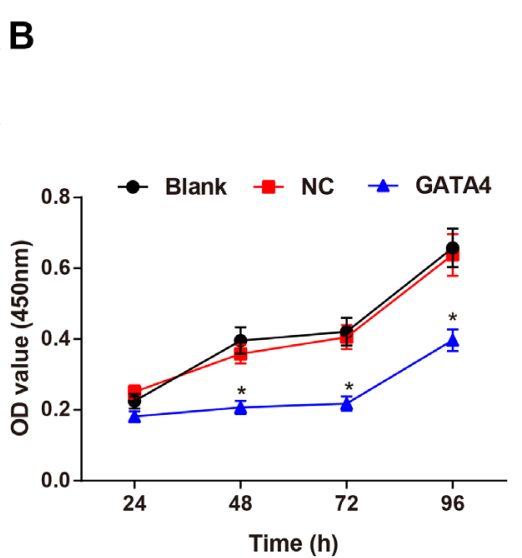

D

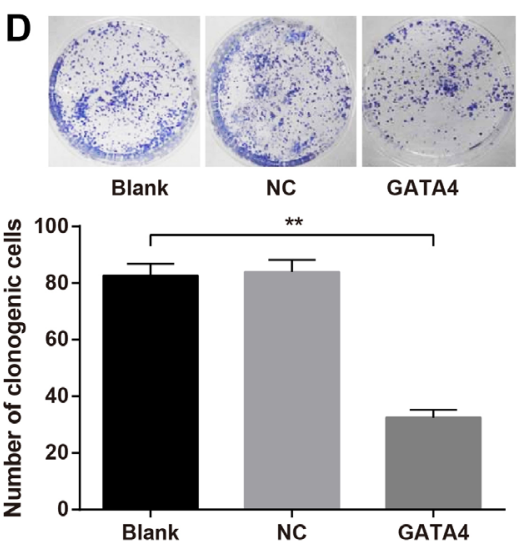

Figure 3. Restoration of GATA4 inhibits the proliferation of SGC-7901 cells. A) GATA4 levels in cells after overexpression of GATA4 were detected by RT-qPCR and western blot analysis; B) CCK-8 assay was used to detect cell proliferation; C) EdU assay was used to detect DNA replication activity; D) colony formation assay was used to detect cell colony formation ability, Repetitions $=3$. Two-way ANOVA was used to analyze the data in panel B, one-way ANOVA was used for data in other panels, and Tukey's multiple comparisons test was used for pairwise comparisons after ANOVA; ${ }^{*} \mathbf{p}<0.05$, compared with the blank group at the same time point; ${ }^{* *} p<0.01$. GC, gastric cancer; RT-qPCR, reverse-transcription quantitative polymerase chain reaction; CCK-8, cell counting kit-8; EdU, 5-ethynyl-2'-deoxyuridine; ANOVA, variance of analysis. 

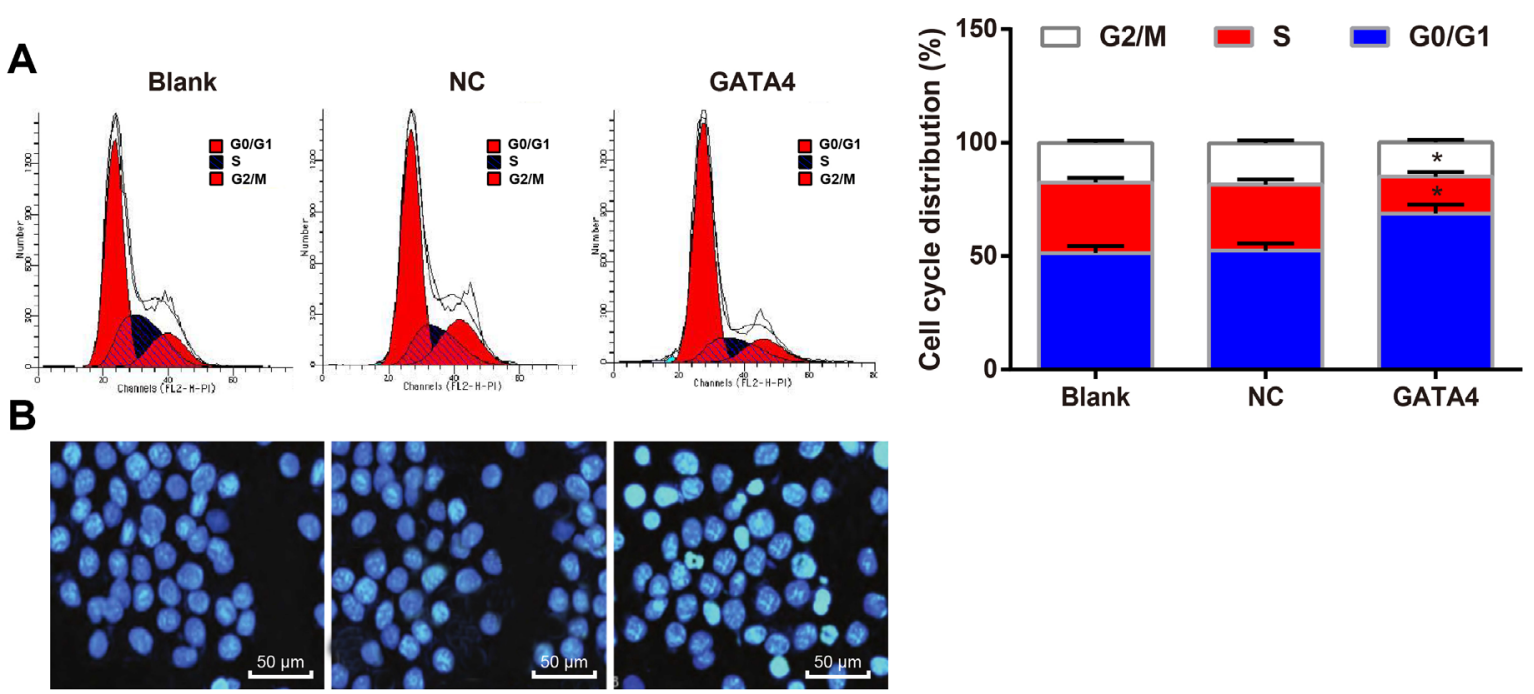

C
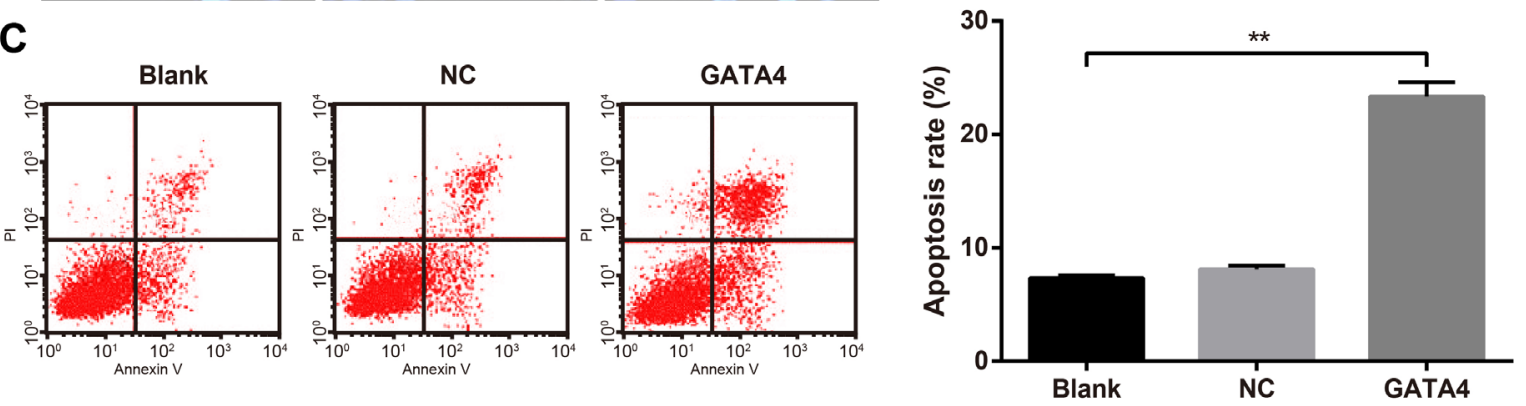

Figure 4. Restoration of GATA4 inhibits SGC-7901 cell cycle and promotes apoptosis. A) Flow cytometry was used to detect cell cycle distribution; B) Hoechst 33258 staining was used to detect cell apoptotic morphology; C) Flow cytometry was used to detect cell apoptotic rate, Repetitions = 3. Twoway ANOVA was used to analyzed data in panel A, one-way ANOVA was used to analyze the data in panel C, and Tukey's multiple comparisons test was used for pairwise comparisons after ANOVA; ${ }^{\star} \mathrm{p}<0.05 ;{ }^{* *} \mathrm{p}<0.01$. GC, gastric cancer; ANOVA, variance of analysis.

results (Figure 6B), suggesting the overexpression of GATA4 could inhibit the expression of key factors in Wnt/ $\beta$-catenin signaling pathway. In conclusion, overexpression of GATA4 may play an anti-GC role by inhibiting $\mathrm{Wnt} / \beta$-catenin signaling pathway.

\section{Discussion}

It is generally recognized that GC is one of the most prevalent malignant tumors in the alimentary canal and is seen as a major health problem due to its high frequency, aggressiveness, and low cure rate [14]. A recent report revealed that GATA3 could serve as an oncogene in GC development and potentially act as a novel therapeutic target [15]. But there were few studies about the mechanism of GATA4 in GC development. In this study, we investigate the role of another member of the GATA family - GATA4 on the development of GC. Consequently, we found that the GATA4 overexpression inhibited GC cell proliferation and promoted cell apoptosis.

Initially, MS-HRM was performed to detect the methylation level of the GATA4 promoter region in GC tissues and cells, finding that the methylation rates of the GATA4 promoter region in GC tissues and cells were significantly higher than those in adjacent normal tissues and human normal gastric mucosa cell line GES-1. This indicated that there was abnormal methylation of GATA4 in GC. Tumor cell-specific promoter hypermethylation, which exerts critical functions on regulating cell cycle, apoptosis, and differentiation, is a common characteristic of cancer [16]. It was reported that promoter hypermethylation induced loss of the GATA4 expression in colorectal, gastric, and esophageal cancer [17]. Additionally, after the detection of GATA4 levels by RT-qPCR and western blot analysis, we found that GATA4 was lowly expressed in GC tissues and cells, and levels of GATA4 in GC were regulated by the methylation of its promoter region, and the abnormal methylation of GATA4 led to the low level of GATA4. GATA4 was also found to be abruptly reduced or even absent in ovarian cancer, and its reduction was supposed to explain the dedifferentiation of ovarian cancer cells [18]. As a former study demonstrated, downregulating gene expression via DNA hypermethylation can often be associated with poor clinical outcome in several malignancies [16]. In a very recent study, it was revealed that the expression of GATA4 was repressed in GC tissues and 
A
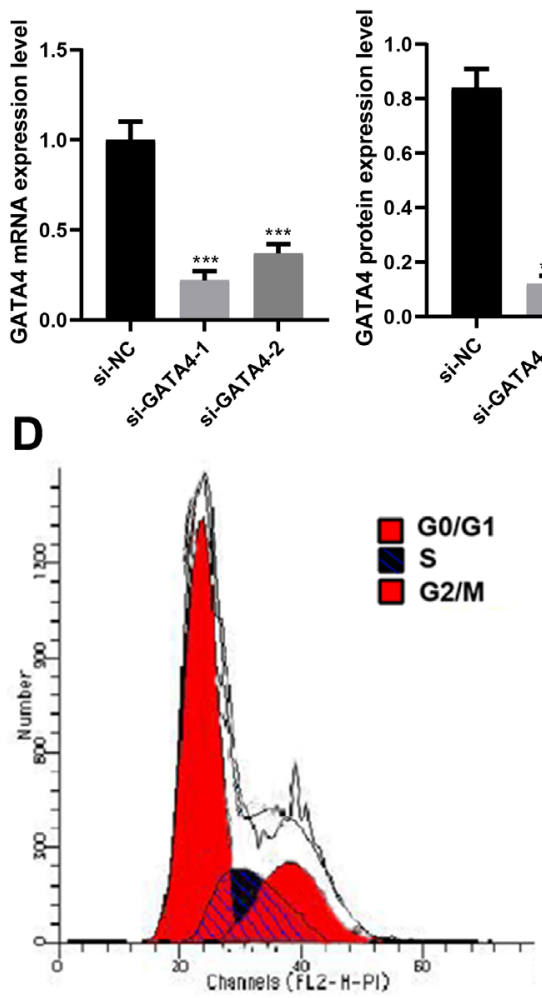

$\mathbf{E}$

B
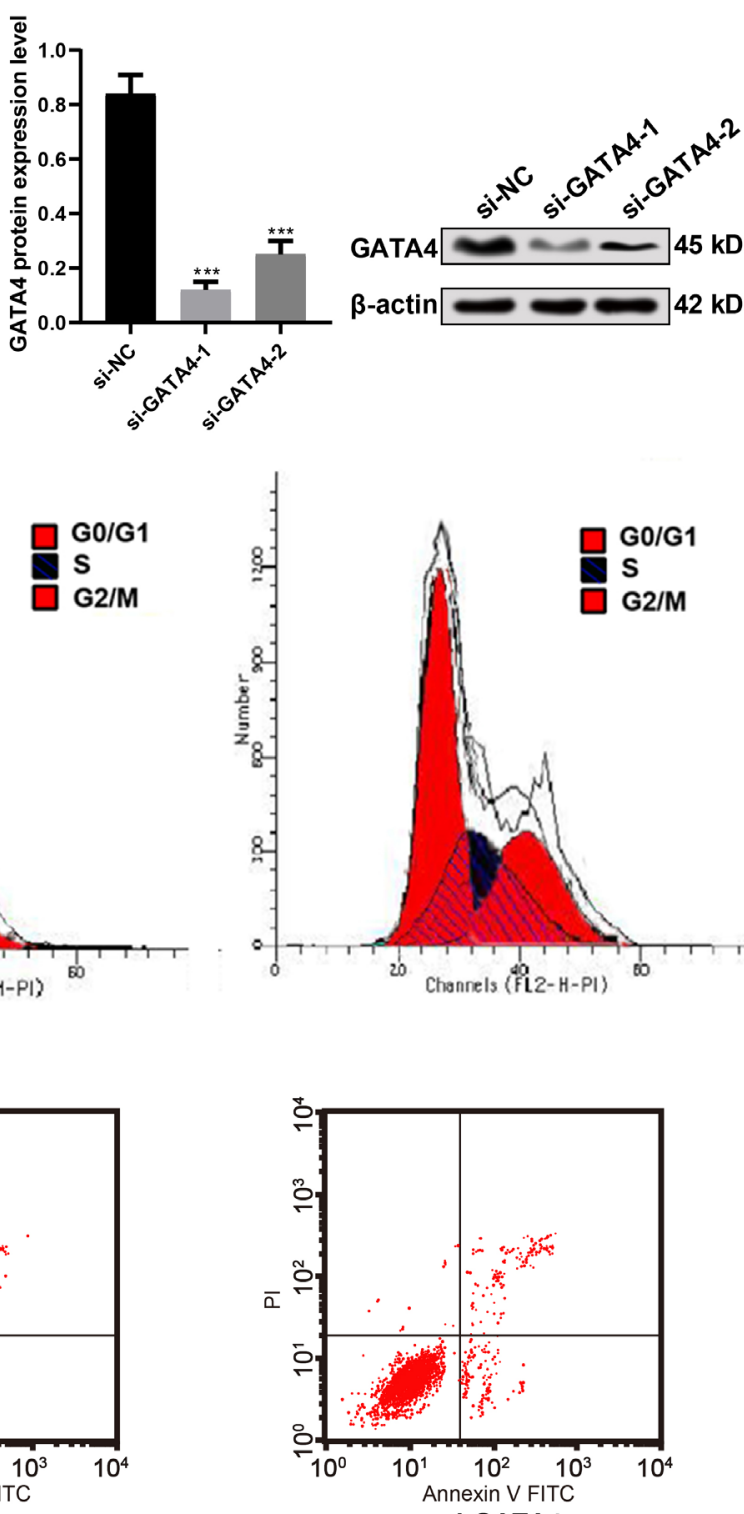

si-GATA4
C
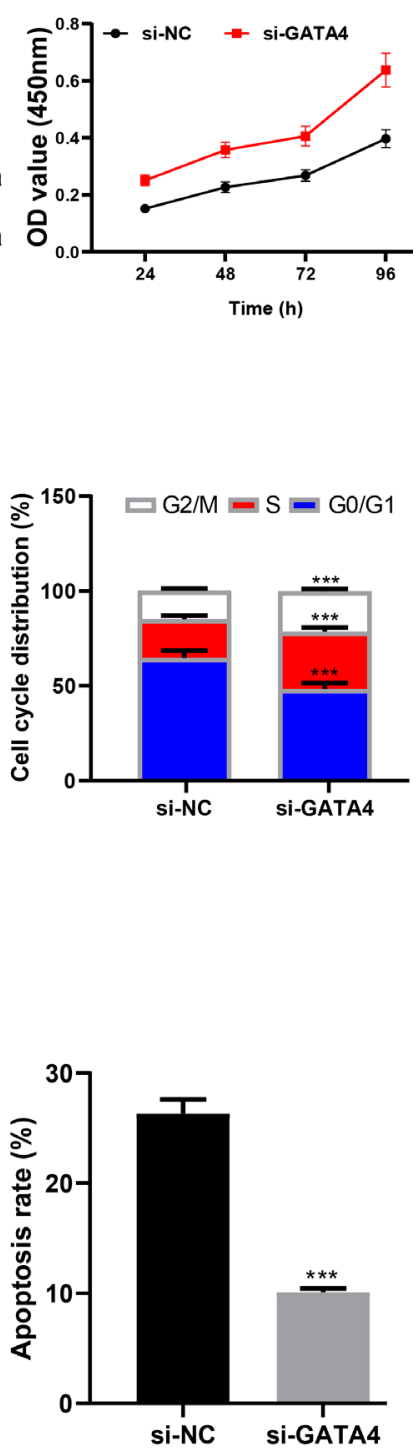
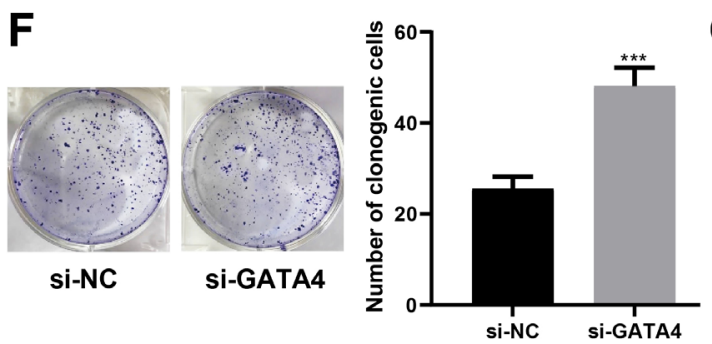

G
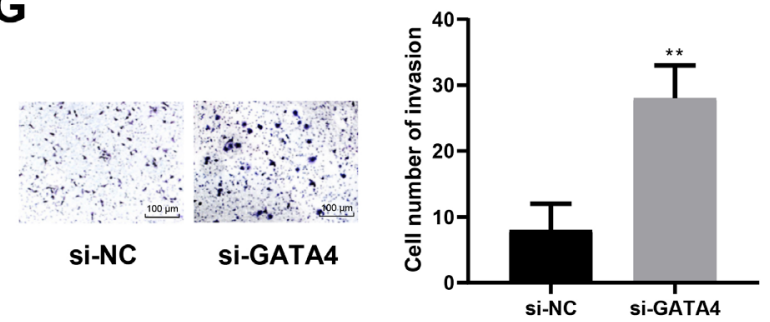

Figure 5 Low expression of GATA4 in GES-1 cells promotes cell migration and increases cell apoptosis. A) mRNA expression of GATA4 detected by RTqPCR; B) protein level of GATA4 detected by western blot analysis; C) Cell cycle distribution detected by flow cytometry; D) Cell apoptosis detected by flow cytometry; E) Clone-forming ability measured by colony formation assay; F) Cell migration ability measured by Transwell assay; Repetitions = 3. Data in panel C and D were analyzed by two-way ANOVA, in other panels analyzed by one-way ANOVA, and pairwise comparison after ANOVA was conducted by Tukey's multiple comparisons test. ${ }^{* *} \mathrm{p}<0.01,{ }^{* * *} \mathrm{p}<0.001$. RT-qPCR, reverse-transcription quantitative polymerase chain reaction; ANOVA, variance of analysis. 


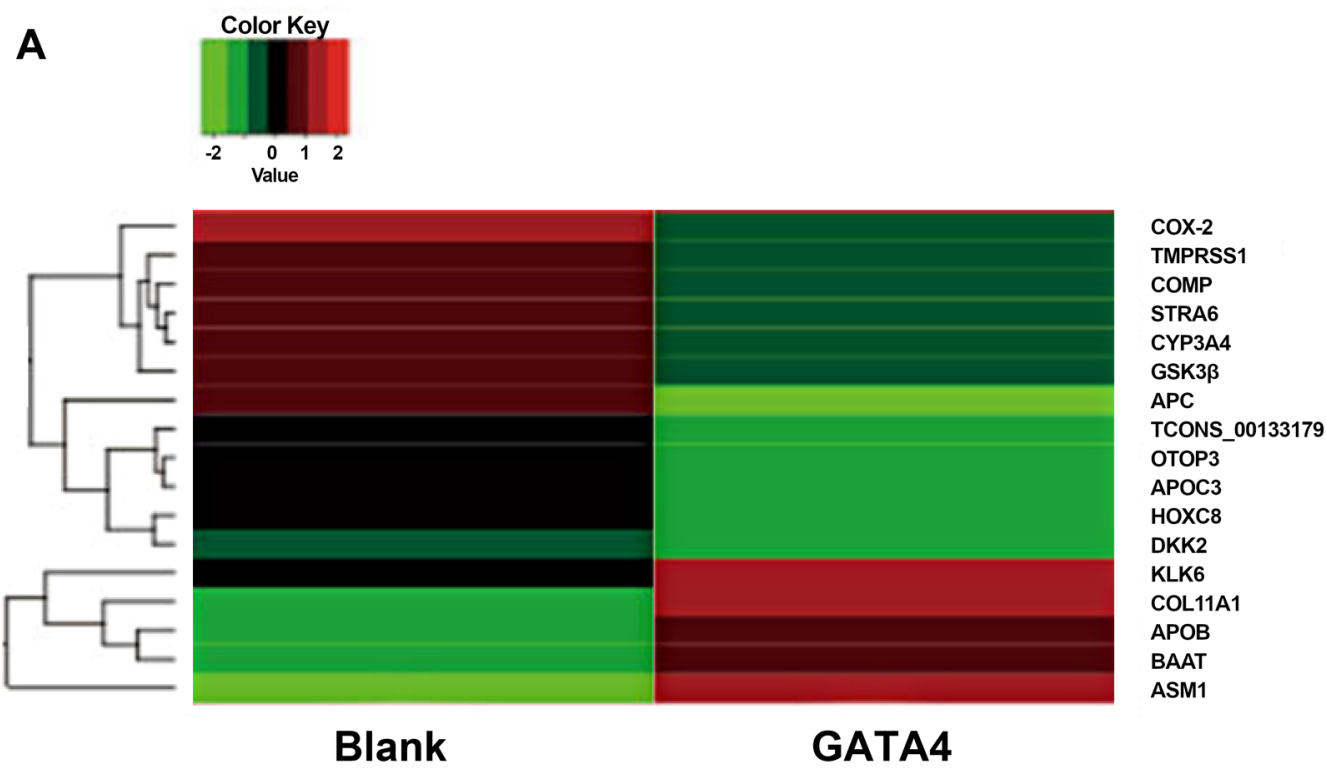

B
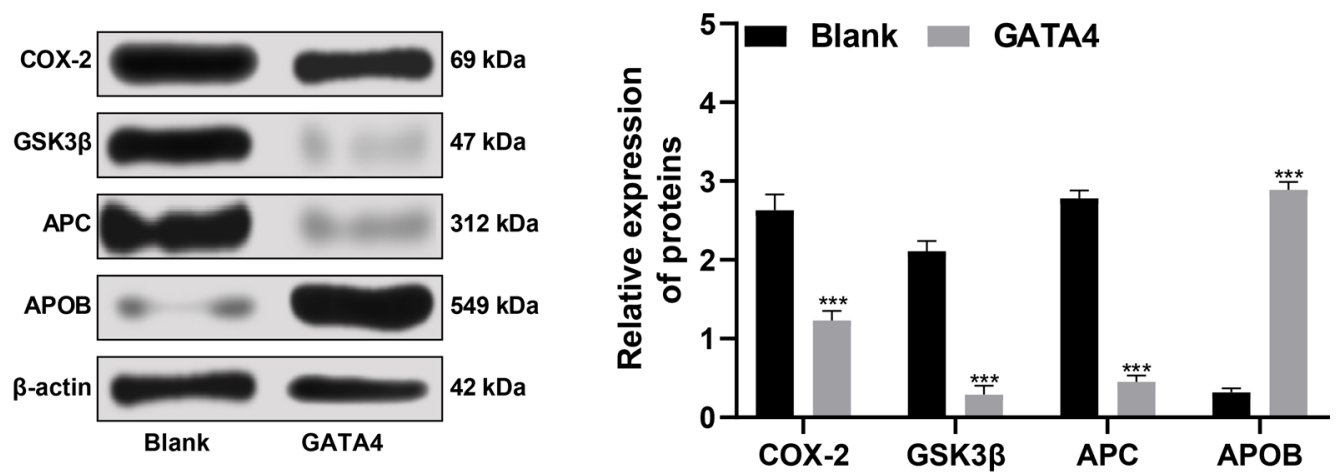

Figure 6. Overexpression of GATA4 plays an anti-GC role by inhibiting Wnt/ $\beta$-catenin signaling pathway. A. Differentially expressed mRNAs analyzed by RNA-seq; protein levels of differentially expressed mRNAs measured by western blot analysis. Repetitions = 3. Data were analyzed by two-way ANOVA, and pairwise comparison after ANOVA was conducted by Tukey's multiple comparisons test. Compared with the GATA4 group, ${ }^{\star \star \star}$ p $<0.001$. GC, gastric cancer; ANOVA, variance of analysis.

cell lines [19], which was consistent with our result that the downregulation of GATA4 in GC was induced by hypermethylation.

Moreover, the relationship between the GATA4 methylation and the clinical characteristics of GC patients was analyzed. Our results revealed that the GATA4 methylation was related to the clinical stage, lymph node metastasis, and tumor differentiation. Interestingly, research mainly published by Wei et al. showed that the expression of GATA3 was associated with tumor size, stage, and metastasis in GC, but there were no correlations between the expression of GATA3 and gender, age, pathological grade of cancer and alcohol consumption of patients [15], which was highly in line with our findings. Our work strongly demonstrated that GATA4 played a significant role in GC development and
GATA4 methylation may be a potential marker for early diagnosis of GC.

Because cell proliferation, colony formation ability and cell apoptosis are crucial biological features in cancer development, we further performed CCK-8, EdU assay, colony formation assay, flow cytometry, and Hoechst 33258 staining to detect the effects of GATA4 on these cell biological processes. The results indicated that the restoration of GATA3 suppressed GC cell proliferation, DNA replication ability and colony formation ability, arrested more cells at G0/G1 phase, and promoted cell apoptosis rate possibly through Wnt/ $\beta$-catenin signaling pathway. GATA4 silencing reduced the proliferation of YCC3 cells, one kind of GC cells [10]. Restoration of GATA3 suppressed GC cell proliferation, migration, and invasion [15]. Besides, the GATA4 overex- 
pression had been reported to repress colony formation, proliferation, migration, and invasion abilities of colorectal cancer cells, thus exerting tumor suppressive effects in colorectal cancer cells in vitro [20]. What's more, restoration of the GATA4 expression was also found to inhibit proliferation and colony formation ability, as well as induce apoptosis of hepatocellular carcinoma cells [21]. Sawada et al. showed that the increased activation of the Wnt/ $\beta$-catenin pathway was associated with poor survival in GC patients [22].

In conclusion, our data suggested that the GATA4 overexpression induced by 5-Aza treatment inhibited GC cell proliferation, colony formation ability, and promoted cell apoptosis via the regulation of its gene promoter methylation and possibly through $\mathrm{Wnt} / \beta$-catenin signaling pathway. This study may provide a new target for the prevention and treatment of GC. However, future studies are required to verify our findings and to clarify the exact mechanisms of GATA4 in GC.

\section{References}

[1] WANG X, LIU B, WEN F, SONG Y. MicroRNA-454 inhibits the malignant biological behaviours of gastric cancer cells by directly targeting mitogen-activated protein kinase 1. Oncol Rep 2018; 39: 1494-1504. https://doi.org/10.3892/ or.2017.6171

[2] FERLAY J, SHIN HR, BRAY F, FORMAN D, MATHERS C et al. Estimates of worldwide burden of cancer in 2008: GLOBOCAN 2008. Int J Cancer 2010; 127: 2893-2917. https:// doi.org/10.1002/ijc.25516

[3] JING JJ, LIU HY, HAO JK, WANG LN, WANG YP et al. Gastric cancer incidence and mortality in Zhuanghe, China, between 2005 and 2010. World J Gastroenterol 2012; 18: 1262-1269. https://doi.org/10.3748/wjg.v18.i11.1262

[4] COMPARE D, ROCCO A, NARDONE G. Risk factors in gastric cancer. Eur Rev Med Pharmacol Sci 2010; 14: 302308.

[5] CORREA P. Gastric cancer: overview. Gastroenterol Clin North Am 2013; 42: 211-217. https://doi.org/10.1016/j. gtc.2013.01.002

[6] WANG CY, HUA L, YAO KH, CHEN JT, ZHANG JJ et al. Long non-coding RNA CCAT2 is up-regulated in gastric cancer and associated with poor prognosis. Int J Clin Exp Pathol 2015; 8: 779-785.

[7] ZHENG R, BLOBEL GA. GATA Transcription Factors and Cancer. Genes Cancer 2010; 1: 1178-1188. https://doi. org/10.1177/1947601911404223

[8] BEULING E, BAFFOUR-AWUAH NY, STAPLETON KA, ARONSON BE, NOAH TK et al. GATA factors regulate proliferation, differentiation, and gene expression in small intestine of mature mice. Gastroenterology 2011; 140: 1219-1229. e1-2. https://doi.org/10.1053/j.gastro.2011.01.033

[9] SUZUKI YJ. Cell signaling pathways for the regulation of GATA4 transcription factor: Implications for cell growth and apoptosis. Cell Signal 2011; 23: 1094-1099. https://doi. org/10.1016/j.cellsig.2011.02.007
[10] CHIA NY, DENG N, DAS K, HUANG D, HU L et al. Regulatory crosstalk between lineage-survival oncogenes KLF5, GATA4 and GATA6 cooperatively promotes gastric cancer development. Gut 2015; 64: 707-719. https:/doi. org/10.1136/gutjnl-2013-306596

[11] MEEK PM, SOOD A, PETERSEN H, BELINSKY SA, TESFAIGZI Y. Epigenetic change (GATA-4 gene methylation) is associated with health status in chronic obstructive pulmonary disease. Biol Res Nurs 2015; 17: 191-198. https://doi. org/10.1177/1099800414538113

[12] CHMELAROVA M, DVORAKOVA E, SPACEK J, LACO J, PALICKA V. Importance of promoter methylation of GATA4 gene in epithelial ovarian cancer. Biomed Pap Med Fac Univ Palacky Olomouc Czech Repub 2013; 157: 294-297. https:// doi.org/10.5507/bp.2013.079

[13] LU H, HUANG S, ZHANG X, WANG D, ZHANG X et al. DNA methylation analysis of SFRP2, GATA4/5, NDRG4 and VIM for the detection of colorectal cancer in fecal DNA. Oncol Lett 2014; 8: 1751-1756.https://doi.org/10.3892/ol.2014.2413

[14] PATRU CL, SURLIN V, GEORGESCU I, PATRU E. Current issues in gastric cancer epidemiology. Rev Med Chir Soc Med Nat Iasi 2013; 117: 199-204.

[15] WEI S, ZHONG L, WANG X, ZHANG W. Low expression of GATA3 promotes cell proliferation and metastasis in gastric cancer. Cancer Manag Res 2017; 9: 769-780. https://doi. org/10.2147/CMAR.S147973

[16] AZHIKINA T, KOZLOVA A, SKVORTSOV T, SVERDLOV E. Heterogeneity and degree of TIMP4, GATA4, SOX18, and EGFL7 gene promoter methylation in non-small cell lung cancer and surrounding tissues. Cancer Genet 2011; 204: 492-500. https://doi.org/10.1016/j.cancergen.2011.07.010

[17] VAITKIENE P, SKIRIUTE D, SKAUMINAS K, TAMASAUSKAS A. GATA4 and DcR1 methylation in glioblastomas. Diagn Pathol 2013; 8: 7. https://doi.org/10.1186/1746-1596-8-7

[18] CAI KQ, CASLINI C, CAPO-CHICHI CD, SLATER C, SMITH ER et al. Loss of GATA4 and GATA6 expression specifies ovarian cancer histological subtypes and precedes neoplastic transformation of ovarian surface epithelia. PLoS One 2009; 4: e6454. https://doi.org/10.1371/journal. pone.0006454

[19] YAO Q, GU A, WANG Z, XUE Y. MicroRNA-144 functions as a tumor suppressor in gastric cancer by targeting cyclooxygenase-2. Exp Ther Med 2018; 15: 3088-3095. https://doi. org/10.3892/etm.2018.5763

[20] HELLEBREKERS DM, LENTJES MH, VAN DEN BOSCH SM, MELOTTE V, WOUTERS KA et al. GATA4 and GATA5 are potential tumor suppressors and biomarkers in colorectal cancer. Clin Cancer Res 2009; 15: 3990-3997. https://doi. org/10.1158/1078-0432.CCR-09-0055

[21] XIA L, GONG Y, ZHANG A, CAI S, ZENG Q. Loss of GATA5 expression due to gene promoter methylation induces growth and colony formation of hepatocellular carcinoma cells. Oncol Lett 2016; 11: 861-869. https://doi.org/10.3892/ ol.2015.3974

[22] SAWADA G, UEO H, MATSUMURA T, UCHI R, ISHIBASHI $\mathrm{M}$ et al. CHD8 is an independent prognostic indicator that regulates Wnt/beta-catenin signaling and the cell cycle in gastric cancer. Oncol Rep 2013; 30: 1137-1142. https:// doi.org/10.3892/or.2013.2597 